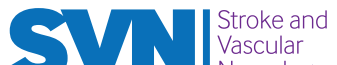
Neurology

\title{
national effort to improve healthcare quality for acute stroke and transient ischaemic attack: rationale, design and preliminary findings
}

Yongjun Wang, ${ }^{1,2,3,4,5}$ Zixiao Li, ${ }^{1,3}$ Yilong Wang, ${ }^{1,2}$ Xingquan Zhao, ${ }^{1,3}$ Liping Liu, ${ }^{1,4,5,6}$ Xin Yang, ${ }^{1,2,3}$ Caiyun Wang, ${ }^{1}$ Hongqiu Gu, ${ }^{1,2,3}$ Fuying Zhang, ${ }^{7}$ Chunjuan Wang, ${ }^{1,2,3,4}$ Ying Xian, ${ }^{8}$ David Z Wang, ${ }^{9}$ Qiang Dong, ${ }^{10}$ Anding Xu, ${ }^{11}$ Jizong Zhao, ${ }^{1,12}$ on behalf of Chinese Stroke Center Alliance investigators

To cite: Wang Y, Li Z, Wang Y, et al. Chinese Stroke Center Alliance: a national effort to improve healthcare quality for acute stroke and transient ischaemic attack: rationale, design and preliminary findings. Stroke and Vascular Neurology 2018;3: e000154. doi:10.1136/ svn-2018-000154

- Additional material is published online only. To view please visit the journal online (http://dx.doi.org/).

Received 20 March 2018 Revised 31 July 2018 Accepted 3 August 2018 Published Online First 8 September 2018

\section{Check for updates}

(C) Author(s) (or their employer(s)) 2018. Re-use permitted under CC BY-NC. No commercial re-use. See rights and permissions. Published by BMJ.

For numbered affiliations see end of article.

Correspondence to Professor Yongjun Wang; yongjunwang1962@gmail.com

\section{ABSTRACT}

Background In June 2015, the Chinese Stroke Association (CSA) initiated the Chinese Stroke Center Alliance (CSCA) to establish the national hospital-based stroke care quality assessment and improvement platform. This article outlines its objectives, operational structure, patient population, quality improvement (Ql) intervention tools, data elements, data collection methodology and current patient and hospital data.

Methods The CSCA is a national, hospital-based, multicentre, voluntary, multifaceted intervention and continuous Ql initiative. This multifaceted intervention includes stroke centre development, written care protocols, workshops and a monitoring/feedback system of evidencebased performance measures. The data coordinating centre of the CSCA resides at the China National Clinical Research Center for Neurological Diseases, Beijing Tiantan Hospital.

Results As of July 2017, 1576 hospitals in China have contributed detailed clinical information to serve as a benchmark for the stroke care quality of 433264 patients with acute stroke/transientischaemic attacks (TIA), including 352572 (81.38\%) acute ischaemic stroke, 30362 (7.01\%) TIA, 42080 (9.71\%) spontaneous intracranial haemorrhage, $5505(1.27 \%)$ subarachnoid haemorrhage and $2745(0.63 \%)$ not specified stroke.

Conclusion The CSCA programme is designed to establish a continuous national stroke registry and help healthcare providers develop stroke centres and treat patients in a consistent manner in accordance with accepted national guidelines and, ultimately, improve patient outcomes. It supports the CSA mission to reduce stroke burden in China.

\section{INTRODUCTION}

Stroke has become an enormous health issue across the world. ${ }^{12}$ Stroke burden in China has increased over the past three decades. More than 10 million Chinese are living with stroke with 2.4 million new cases in 2013. ${ }^{3}$ Improving healthcare quality is full of challenges but imperative. ${ }^{4}$ Over the past decade, stroke centre construction and quality improvement (QI) initiatives, such as the Get With The Guidelines-Stroke (GWTGStroke) programme and Canadian Best Practice Recommendations for Stroke Care, have been implemented to significantly improve stroke care quality and outcomes in those high-income countries. ${ }^{5-10}$ Designated stroke centres have increased the use of thrombolytic therapy and modestly decreased mortality of hospitalised patients with acute ischaemic stroke (IS). ${ }^{5}$ Systems of care have to be structured efficiently to facilitate the delivery of care. ${ }^{1}$

Over the last decade, significant changes in the Chinese healthcare system have occurred with universal health insurance coverage in 2011. ${ }^{11}$ Stroke care improvement has become a national priority. Since 2007, several national strategies have been adopted to establish national stroke research networks and stroke registries, increase the compliance with evidence-based process performance measures of stroke care and develop stroke care organisation. ${ }^{12-14}$ In addition, clinical research and evidence-based guidelines for stroke and transient ischaemic attacks (TIA) care, with advanced diagnostic and treatment modalities, have been developed in China. ${ }^{15-17}$

These abovementioned national strategies in the healthcare system, policy and clinical research have improved, to some degree, evidence-based stroke care and patient outcomes. ${ }^{14}$ However, a comparison of two China National Stroke Registries (2007-2008 vs 2012-2013) demonstrated that adherence to these evidence-based interventions remains suboptimal, and gaps in adherence to 
guideline-recommended care are even greater in China when compared with other high-income counties, such as USA. ${ }^{81418}$ For instance, in 2012, adherence to intravenous tissue plasminogen activator use $(18.3 \%)$ and anticoagulation for atrial fibrillation (21.0\%) is still low in China and was far below 2007 GWTG-Stroke adherence $(72.84 \%$ and $98.39 \%$, respectively). ${ }^{814}$ In 2012 , a stroke centre survey of 521 hospitals from the China Stroke Research Network demonstrated that only $20(3.8 \%)$ met the criteria for a comprehensive stroke centre, and only 179 (34.4\%) met the criteria for a primary stroke centre. ${ }^{19}$ A multifaceted QI intervention increased hospital personnel adherence to evidence-based performance measures in patients with acute IS in China. ${ }^{20}$

To bridge these remarkable gaps between guideline recommendations and clinical practices, the Chinese Stroke Center Alliance (CSCA) programme was launched by the Chinese Stroke Association (CSA) in 2015. This article introduces CSCA's objectives, operational structure, patient population, QI intervention tools, data elements, data collection methodology and current patient and hospital data.

\section{OBJECTIVES}

(1) Promote stroke centre development and organise the delivery of stroke care; (2) function as a national registry system to evaluate the features, managements and outcomes of patients hospitalised with acute stroke/TIA; (3) improve the management and outcomes of patients with acute stroke/TIA through pragmatic multifaceted QI tools to translate evidence-based guideline recommendations into clinical practice.

\section{METHODS}

\section{CSCA programme design}

The CSCA was designed and led by a team of volunteers from the CSA under the guidance of the National Center of Neurological Diseases Care Management. The CSCA is a national, hospital-based, multicentre, voluntary, multifaceted intervention and continuous QI initiative. This programme is made available to all Chinese secondary and tertiary grade hospitals. Hospitals continued to join the programme in a staggered manner. Hospital characteristics, including geographic region, teaching status, hospital volume (secondary and tertiary grade) and annual stroke volume, are surveyed.

The CSCA was announced by CSA on its website and through the press and social media, such as WeChat. Hospitals intending to join this programme contacted the staff of CSA voluntarily. Hospitals were also recruited directly by regional CSA staff and volunteers by working with the National and/or Provincial Center of Neurological Diseases Care Management. The number of hospitals from each province was abstracted from Statistical Yearbook 2016 published by National Health and Family Planning Commission of the People's Republic of China. ${ }^{21}$

\section{Patient population}

The CSCA requires participating hospitals to enrol consecutive patients who meet the following criteria: (1) are aged 18 years or older; (2) have a primary diagnosis of acute stroke/TIA confirmed by brain CT or MRI, including IS, TIA, intracerebral haemorrhage or subarachnoid haemorrhage $(\mathrm{SAH})$; (3) are within 7 days of symptom onset; (4) are admitted either directly to wards or through the emergency department. Patients with cerebral venous sinus thrombosis or non-cerebrovascular diseases were excluded.

\section{Data collection and management}

Data were collected via the web-based patient data collection and management tool (Medicine Innovation Research Center, Beijing, China), abstracted via chart review, coded, deidentified and transmitted in a secure manner to maintain patient confidentiality compliant with national privacy standards. The following data were collected for each hospitalisation: patient demographics, history of disease and medication, hospital presentation, initial neurological status, medications and interventions, reperfusion strategy and in-hospital outcomes and complications. The detailed content of each category can be found in online supplementary table 1 . The definition of in-hospital outcomes and complications are listed in online supplementary table 2.

This patient data collection and management tool has two main functions. Its first function is to collect concurrent data. The data abstraction tool is characterised by predefined logic features, range checks and user alerts to identify a potentially invalid format or value entries and to optimise data quality at the time of entry. The required items were structured so that valid data must be entered before the data can be saved as a complete record and submitted to the database. Range checks were used to find inconsistent or out-of-range data and prompted users to correct or review data entries that were outside a predefined range. To create an audit trail for data entered or changed, all hospital personnel using the tool received individual passwords. Training in the use of the tool was provided online and onsite for all users. However, data collected by hospitals were not independently audited by external chart review.

The second function of the tool is to analyse and provide data feedback. The China National Clinical Research Center for Neurological Diseases (NCRCND) serves as the data analysis centre and has an agreement to analyse the aggregate deidentified data for care quality feedback and research purposes. All hospitals using the tool received an independent account and password to view the benchmark for adherence to evidence-based performance measures and to compare their own hospital's current performance to past levels and the concurrent standards of other regional hospitals within their purview. Information on stroke care quality was also sent to hospital personnel via the Wechat App, a communication tool. 


\section{Performance metrics adopted by CSCA}

In order to assess quality of stroke care, there are a total of 13 performance metrics of IS based on the national standards and guideline recommendations prespecified or updated by the Steering Committee of CSCA, including six acute performance measures: (1) intravenous recombinant tissue plasminogen activator $<4.5$ hours, (2) endovascular treatment $<6$ hours, (3) early antithrombotics, (4) deep vein thrombosis prophylaxis, (5) dysphagia screening, (6) rehabilitation assessment. There are then seven performance measures at discharge: (1) antithrombotic medication, (2) anticoagulation for atrial fibrillation, (3) antihypertensive medicines for patients with hypertension, (4) statin therapy for low-density lipoprotein $\geq 100 \mathrm{mg} / \mathrm{dL}$, (5) hypoglycaemia medication for diabetes mellitus, (6) smoking cessation and (7) stroke education. The specific definitions of these performance measures are shown in online supplementary table 3 .

\section{Multifaceted quality improvement intervention}

Data collection and performance reporting are an essential and ongoing part of the continuously circumnavigating QI process. Meanwhile, the CSCA primarily focuses on the construction of stroke centres at hospitals according to Chinese guidelines, organisational stakeholder and opinion leader meetings, collaborative workshops and webinars for hospital teams, hospital toolkits and hospital recognition. Data collection, decision support and hospital data feedback via multiple on-demand reports of performance on all key measures were conducted with the web-based patient data collection and management tool. Workshops included the didactic presentation of clinical trial evidence and national or international guidelines for acute treatment and secondary prevention of stroke and TIA, followed by examples of successful hospital implementation. These workshops were given by CSA staff and volunteers with expertise in clinical science and QI. Videos of these workshops were readily available online and via mobile apps.

Stroke centre construction consists of two stages. The first stage is self-assessment by a hospital to identify its degree of compliance with the criteria of a stroke centre, as established by Chinese guideline of stroke centre construction. The second stage is verification by the CSA for compliance with applicable guidelines. Verification is based on a review of information from combined sources, including self-assessment statements, data collection, care quality assessment. Verified hospitals are awarded a 'verified' status that remains valid for 2 years, and they are granted permission to display a 'seal of approval' logo.

\section{CSCA organisation, operations and funding}

Routine operations of the CSCA are conducted and managed by the CSA staff. These operations are as follows: update and maintain the CSCA web-based data collection and management tool; develop and run site feedback reports and execute research analyses as part of its role as the CSCA data coordinating centre. Provincial
Centers of Neurological Diseases/Stroke Care Management (listed in supplemental materials) are responsible for the management of CSCA hospitals in their regions. The CSCA's data coordinating centre resides at the NCRCND, Beijing Tiantan Hospital. There is currently no fee for hospitals to participate in the CSCA.

\section{Patient privacy and informed consent issues}

The primary purpose of the CSCA is to facilitate QI. At the local level, data collection by the site is seen as a QI tool. Participating hospitals received either healthcare quality assessment and research approval to collect data in CSCA without requiring individual patient informed consent under the common rule or a waiver of authorisation and exemption from their Institutional Review Board. Notably, informed consent may lead to a sampling bias, which can jeopardise the validity and generalisability of the database.

The aggregate of CSCA data will be used to support national clinical research. Patient confidentiality will be protected in the following ways: (1) data are stripped of all identifiers before their use in research and (2) the use of data for these purposes is closely overseen by the NCRCND analytic centre.

\section{PRELIMINARY RESULTS}

The CSCA programme was initiated by the CSA in June 2015. Data submission for the CSCA was initiated on 1 August 2015. As of July 2017, 1576 hospitals have participated in this programme and contributed detailed clinical information and benchmark of care quality for 433264 patients with acute stroke/TIA. Of the 1576 hospitals, $612(38.8 \%)$ were secondary hospitals and 452 (28.6\%) were located in East China. Among each province, the distribution and percentage of hospitals joining the CSCA programme in the number of hospitals from Statistical Yearbook 2016 is demonstrated in figure 1.

Of the 433264 patients, 352572 (81.38\%) were diagnosed with acute IS, $30362(7.01 \%)$ with TIA, 42080 $(9.71 \%)$ with spontaneous intracranial haemorrhage, $5505(1.27 \%)$ with SAH and $2745(0.63 \%)$ with not specified stroke. The median patient age is 66 years old, over $60 \%$ of patients were men and $96 \%$ of patients were Han. Patients with IS were more likely to have a history of atrial fibrillation/flutter and dyslipidaemia. The patient group with SAH was younger, had a higher percentage of women and was more likely to use the Emergency Medical Service. Other details can be found in table 1 .

\section{DISCUSSION}

CSCA has enrolled 433264 patients with acute stroke/ TIA from 1576 hospitals. The patient and hospital baseline characteristics were reported. Compared with the previous hospitalised stroke registries, CSCA represents a large-scale effort to translate efficacy into effectiveness in stroke/TIA care for hospitalised patients covering all regions of China (except for Hong Kong, Taiwan and 


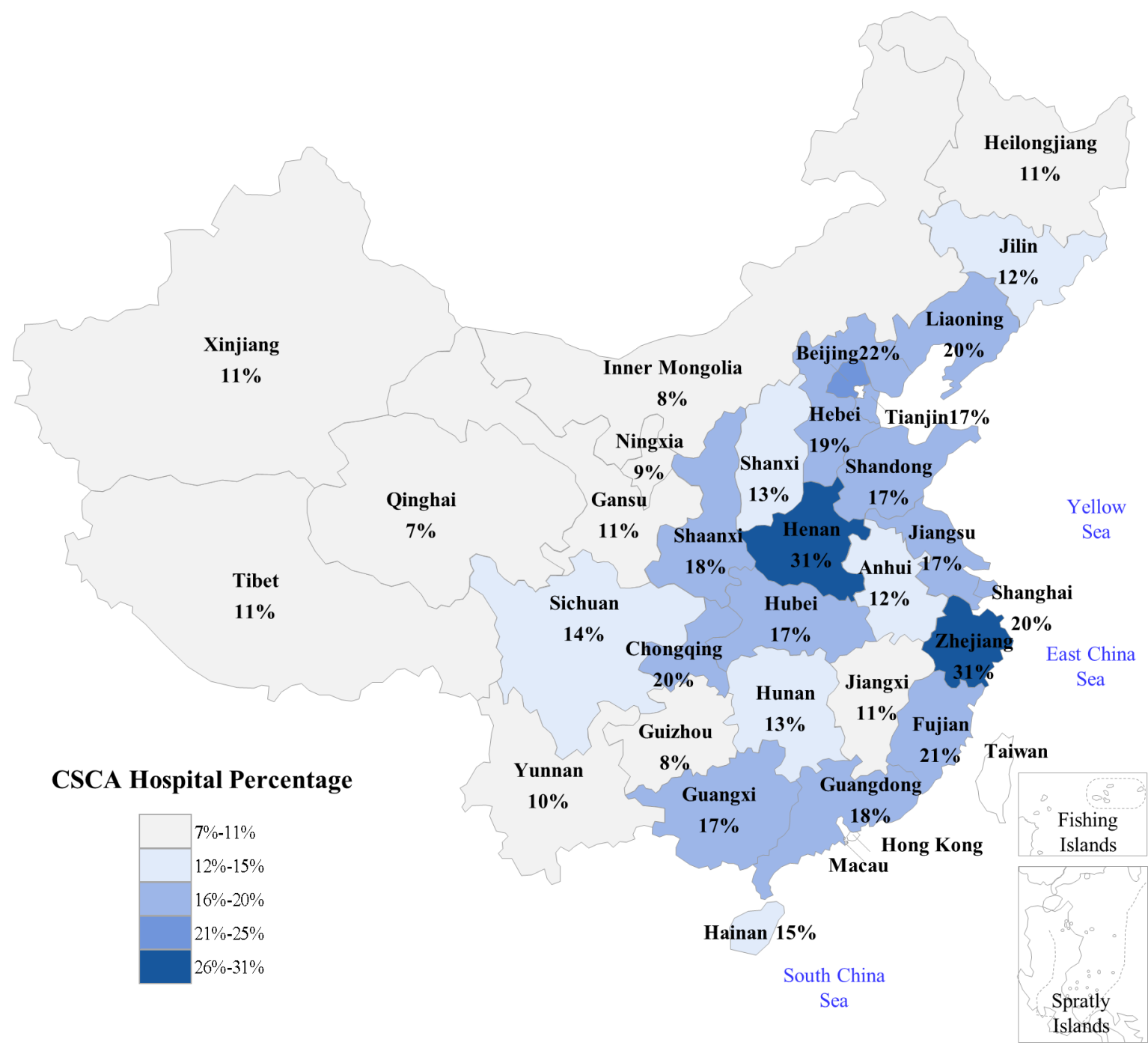

Figure 1 Distribution and percentage of hospitals participating in CSCA programme among all the hospitals each province. CSCA, Chinese Stroke Center Alliance.

Macao regions). Patients with IS from CSCA have the similar patients' demographic and clinical characteristics of China National Stroke Registry (CNSR) I and II, such as patients' age, race, history of stroke/TIA, hypertension disease and diabetes mellitus. ${ }^{14}$ This programme also provides a unique platform to track the characteristics, process of care and outcomes of patients with stroke/TIA. Specifically, the CSCA provides opportunities to promote stroke centre construction and increase adherence to evidence-based performance metric in clinical practice. Through the CSCA, participating hospitals self-assess their stroke centre status and are verified by the CSA. They have access to a robust QI framework, including performance feedback reports and QI tools that are vital to facilitating a quality patient care ambience. The CSCA can also be regarded as a 'test setting' to assess the effectiveness of QI efforts and initiatives. ${ }^{22}$

In addition to playing a role in QI, the CSCA represents the potential for a scientific research resource. First, as a majority of Chinese hospitals participate in this programme, the CSCA can gradually be regarded as a national surveillance system for Chinese acute stroke/ TIA care and patients outcomes. ${ }^{23}$ Second, the CSCA can offer the clue and rationale for future randomised clinical trials. Specifically, observational comparative effectiveness research from the CSCA can be a source of hypothesis-generating, helping to find vital questions for trial verification as well as offering needed information for trial design and sample calculation. ${ }^{22}$ The CSCA could also potentially function as a clinical research network for conducting RCTs, linking interested investigators and limiting the demands of additional data collection. Third, the CSCA can become a platform for tracking and assessing new drugs or devices in routine clinical practice. Such postmarket assessment information is necessary to monitor the effectiveness and safety of these therapeutic agents outside of selected trial population and settings. ${ }^{24}$

Finally, there exist potentials to link CSCA data with other administrative data sources (such as the National Death Cause Registry Database, China Health and Nutrition Survey dataset ${ }^{25}$ ) to provide information on 


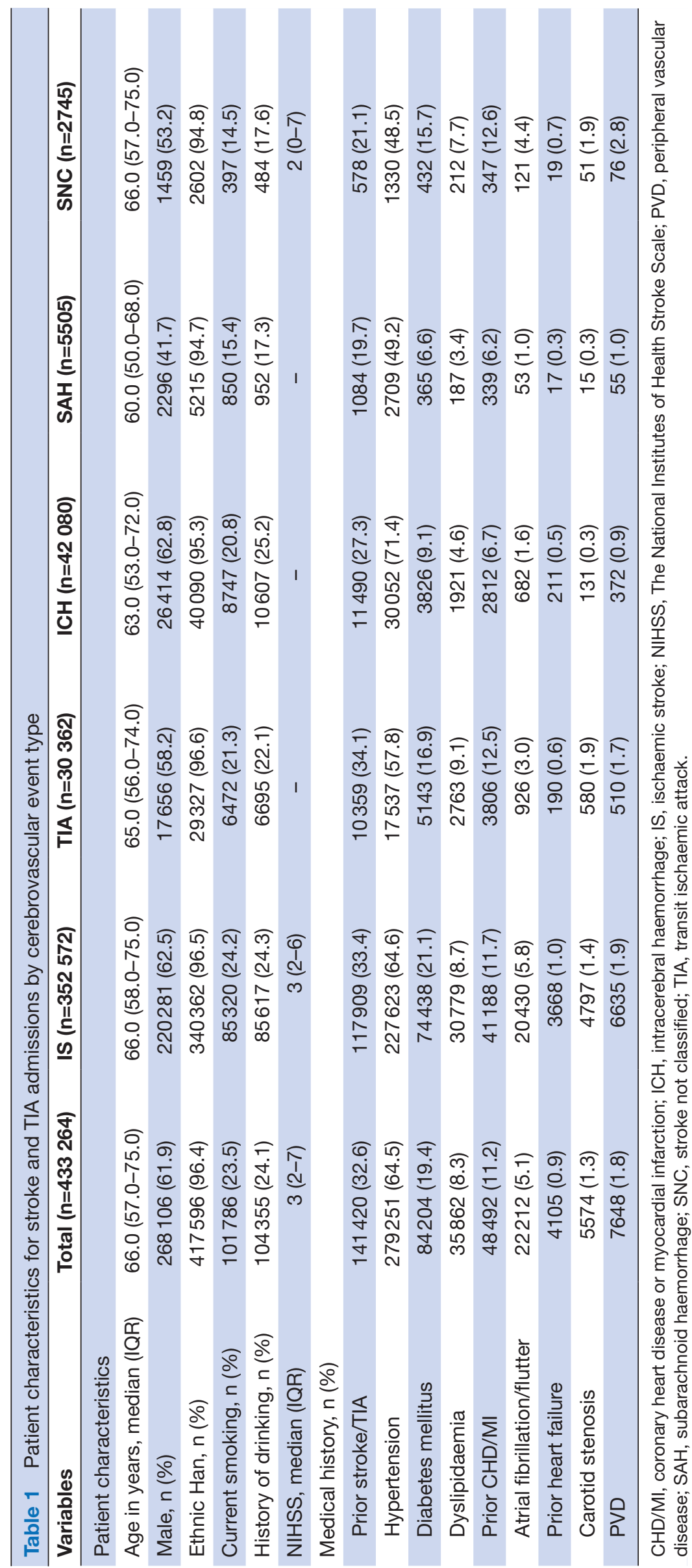


downstream clinical events, such as death. This effort brings increasing challenges of patient privacy, sampling bias and technology.

There are several limitations in the CSCA programme. It must be pointed out that hospital participation in the CSCA is voluntary. As a result, the current participating hospitals are more likely to be larger, tertiary centres with a myriad of resources to which smaller hospitals do not have access. Although hospital recruitment remains ongoing, we recognise that the CSCA may not apply to patients who present with acute stroke/TIA to hospitals outside of the CSCA. Increasing the number of hospitals participating in the CSCA will optimise the representation of hospitals, patients and regions. Second, approximately 1575 of the nation's 9617 public secondary and tertiary hospitals are currently participating in CSCA, so there is huge room for expansion. The means to collect data, as well as limitation of resources, are still obstacles that need to be overcome. Third, data elements in the CSCA are limited to those that are readily documented in the inpatient medical record. Inpatient data in the CSCA lack follow-up information. Additionally, data collected by hospitals were not independently audited by external chart review. Data reliability depends on training for data abstractors and built-in automated checks to identify erroneous, illogical data entries.

There will be several plans to enhance the CSCA programme. First, continuing education for data abstractors will be conducted to enhance the data reliability. Data reliability study will be performed within this QI initiative. Second, the CSCA programme will continue to invite public secondary and tertiary hospitals to participate. Third, a series of training workshops of stroke centre development and stroke care will be continuously held to improve stroke centre construction and stroke care quality. Additionally, the CSCA database will be regarded as a national surveillance system and scientific research resource for Chinese acute stroke/ TIA care and patients outcomes, which will be provided for researchers to conduct stroke care studies.

\section{CONCLUSION}

The CSCA is a national, hospital-based, voluntary, multifaceted intervention and continuous QI initiative and provides a unique platform to develop stroke centres and improve stroke care quality and clinical outcomes. The CSCA will be able to broaden the QI field by building a rich set of data for stroke/TIA surveillance, tracking the usage of evidence-based therapies into clinical practice and comparing the efficiency of different treatments. Additionally, the CSCA will likely be linked with other administrative and clinical databases to conduct a longitudinal assessment of patients with acute stroke/TIA. These contributions will support the CSA mission to reduce stroke recurrence, mortality and disability in China.
Author affiliations

${ }^{1}$ China National Clinical Research Center for Neurological Diseases, Beijing, China ${ }^{2}$ Tiantan Clinical Trial and Research Center for Stroke, Department of Neurology,

Beijing Tiantan Hospital, Capital Medical University, Beijing, China

${ }^{3}$ Vascular Neurology, Department of Neurology, Beijing Tiantan Hospital, Capital Medical University, Beijing, China

${ }^{4}$ Center for Stroke, Beijing Institute for Brain Disorders, Beijing, China

${ }^{5}$ Beijing Key Laboratory of Translational Medicine for Cerebrovascular Disease,

Beijing, China

${ }^{6}$ Neuro-intensive Care Unit, Department of Neurology, Beijing Tiantan Hospital, Capital Medical University, Beijing, China

${ }^{7}$ Office of Chinese Stroke Center Alliance, Chinese Stroke Association, Beijing, China ${ }^{8}$ Department of Neurology, Duke University Medical Center, Durham, North Carolina, USA

${ }^{9}$ INI Stroke Network, OSF Healthcare System, University of Illinois College of Medicine, Peoria, Illinois, USA

${ }^{10}$ Department of Neurology, Shanghai Huashan Hospital, Fudan University, Shanghai, China

${ }^{11}$ Department of Neurology, The First Affiliated Hospital of Jinan University, Guangzhou, China

${ }^{12}$ Department of Neurosurgery, Beijing Tiantan Hospital, Capital Medical University, Beijing, China

Acknowledgements We thank all the participating centres in CSCA programme for their hard work in data collection.

Collaborators Yongjun Wang, Zixiao Li, Yilong Wang, Xingquan Zhao, Liping Liu, Xin Yang, Caiyun Wang, Hongqiu Gu, Fuying Zhang, Chunjuan Wang, Ying Xian, David Wang, Qiang Dong, Anding Xu, Jizong Zhao, Kai Wang, Kai Wang, Xunming Ji, Xinyue Qin, Ning Wang, Zhaoming Ge, Jinsheng Zeng, Lvli Li, Lan Chu, Zhibin Chen, Li Guo, Guozhong Li, Yuming Xu, Bo Hu, Beisha Tang, Guorong Liu, Xiaoshan Wang, Xiaomu Wu, Yi Yang, Zhiyi He, Zhenhai Wang, Shizheng Wu, Gang Zhao, Meijia Zhu, Qiang Dong, Xiaoyuan Niu, Dong Zhou, Zhongping An, Yuhua Zhao, Xiaoning Zhang, Li Ding, Min Lou.

Contributors YoW, ZL, YiW, XZ, LL, YX, DZW, QD, AX and JZZ conceived the study and oversaw all scientific aspects of its implementation. YoW, ZL and HG drafted the manuscript. HG conducted the data analysis. XY, CaW, ChW and FZ recruited hospitals and operated the CSCA programme.

Funding This work was supported by grants from the Ministry of Science and Technology and the Ministry of Health of the People's Republic of China (National S \& T Major Project of China: 2011BAI08B02, 2012ZX09303, 2013BAI09B14, 2013BAI09B03, 2015BAl12B02, 2015BAl12B04, 2017YFC1310900, 2017YFC1310901 and 2017YFC1310903), the National Key Technology Research and Development Program of the Ministry of Science and Technology of China (2013BAI09B03), Beijing Municipal Committee of Science and Technology (D15110700200000, D151100002015001, D151100002015002, Z161100000516223 and Z141107002514125), Beijing Institute for Brain Disorders (BIBD-PXM2013_014226_07_000084).

Competing interests None declared.

Ethics approval Participating hospitals received either healthcare quality assessment and research approval to collect data in the CSCA project without requiring individual patient informed consent under the common rule or a waiver of authorization and exemption from subsequent review by their Institutional Review Board.

Provenance and peer review Not commissioned; externally peer reviewed. Data sharing statement No additional data are available.

Open access This is an open access article distributed in accordance with the Creative Commons Attribution Non Commercial (CC BY-NC 4.0) license, which permits others to distribute, remix, adapt, build upon this work non-commercially, and license their derivative works on different terms, provided the original work is properly cited, appropriate credit is given, any changes made indicated, and the use is non-commercial. See: http://creativecommons.org/licenses/by-nc/4.0/.

\section{REFERENCES}

1. GBD 2016 Causes of Death Collaborators. Global, regional, and national age-sex specific mortality for 264 causes of death, 19802016: a systematic analysis for the Global Burden of Disease Study 2016. Lancet 2017;390:1151-210. 
2. Feigin VL, Forouzanfar MH, Krishnamurthi R, et al. Global and regional burden of stroke during 1990-2010: findings from the Global Burden of Disease Study 2010. Lancet 2014;383:245-55.

3. Wang W, Jiang B, Sun $\mathrm{H}$, et al. Prevalence, incidence, and mortality of stroke in china: Results from a nationwide population-based survey of 480687 adults. Circulation 2017;135.

4. Cabana MD, Rand CS, Powe NR, et al. Why don't physicians follow clinical practice guidelines? A framework for improvement. JAMA 1999;282-1458-65.

5. Xian Y, Holloway RG, Chan PS, et al. Association between stroke center hospitalization for acute ischemic stroke and mortality. JAMA 2011;305:373-80.

6. Man S, Schold JD, Uchino K. Impact of stroke center certification on mortality after ischemic stroke: The medicare cohort from 2009 to 2013. Stroke 2017:48:2527-33.

7. Ormseth $\mathrm{CH}$, Sheth $\mathrm{KN}$, Saver JL, et al. The American Heart Association's Get With the Guidelines (GWTG)-Stroke development and impact on stroke care. Stroke Vasc Neurol 2017;2.

8. Schwamm LH, Fonarow GC, Reeves MJ, et al. Get With the Guidelines-Stroke is associated with sustained improvement in care for patients hospitalized with acute stroke or transient ischemic attack. Circulation 2009;119:107-15.

9. Blacquiere D, Lindsay MP, Foley N, et al. Canadian stroke best practice recommendations: Telestroke best practice guidelines update 2017. Int J Stroke 2017;12:886-95.

10. Fonarow GC, Zhao X, Smith EE, et al. Door-to-needle times for tissue plasminogen activator administration and clinical outcomes in acute ischemic stroke before and after a quality improvement initiative. JAMA 2014:311:1632-40.

11. Yu H. Universal health insurance coverage for 1.3 billion people: What accounts for China's success? Health Policy 2015;119:1145-52.

12. Liu L, Wang D, Wong KS, et al. Stroke and stroke care in China: huge burden, significant workload, and a national priority. Stroke 2011:42:3651-4.

13. Wang Y, Li Z, Zhao X, et al. Stroke care quality in China: Substantial improvement, and a huge challenge and opportunity. Int J Stroke 2017:12:229-35.
14. Li Z, Wang C, Zhao X, et al. Substantial progress yet significant opportunity for improvement in stroke care in china. Stroke 2016;47:2843-9.

15. Wang YJ, Zhang SM, Zhang $L$, et al. Chinese guidelines for the secondary prevention of ischemic stroke and transient ischemic attack 2010. CNS Neurosci Ther 2012;18:93-101.

16. Wang Y, Liu M, Pu C. 2014 Chinese guidelines for secondary prevention of ischemic stroke and transient ischemic attack. International Journal of Stroke 2017;12:302-20.

17. Wang $\mathrm{Y}$, Wang $\mathrm{Y}$, Zhao $\mathrm{X}$, et al. Clopidogrel with aspirin in acute minor stroke or transient ischemic attack. N Engl J Med 2013;369:11-19.

18. Yang X, Li Z, Zhao X, et al. Use of warfarin at discharge among acute ischemic stroke patients with nonvalvular atrial fibrillation in china. Stroke 2016;47:464-70.

19. Chunjuan Wang ZL, Wang Y, Jiang Y, et al. Abstract t mp75: Facilities available in chinese hospitals from china stroke research network. Stroke 2015;46. ATMP75.

20. Wang Y, Li Z, Zhao X, et al. Effect of a multifaceted quality improvement intervention on hospital personnel adherence to performance measures in patients with acute ischemic stroke in china: A randomized clinical trial. JAMA 2018;320:245.

21. National Health and Family Planning Commission. China's Health And Family Planning Statistical Yearbook 2016. Beijing: Peking Union Medical College Press 2017:15.

22. Peterson ED, Roe MT, Rumsfeld JS, et al. A call to ACTION (acute coronary treatment and intervention outcomes network): a national effort to promote timely clinical feedback and support continuous quality improvement for acute myocardial infarction. Circ Cardiovasc Qual Outcomes 2009;2:491-9.

23. Kroll M, Phalkey RK, Kraas F. Challenges to the surveillance of non-communicable diseases-a review of selected approaches. BMC Public Health 2015;15:1243.

24. Sherman RE, Anderson SA, Dal Pan GJ, et al. Real-world evidence what is it and what can it tell us? N Engl J Med 2016;375:2293-7.

25. Zhang B, Zhai FY, Du SF, et al. The China Health and Nutrition Survey, 1989-2011. Obes Rev 2014;15(Suppl 1):2-7. 